\title{
Surface termination during GaN growth by metal-organic vapor phase epitaxy determined by ellipsometry
}

C. Cobet, ${ }^{*}$ T. Schmidtling, M. Drago, N. Wollschläger, N. Esser, and W. Richter Institut für Festkörperphysik, Technische Universität Berlin, Hardenbergstr. 36, D-10623 Berlin, Germany

R. M. Feenstra

Dept. Physics, Carnegie Mellon University, Pittsburgh, PA 15213, USA

T. U. Kampen

Institut für Physik, Technische Universität Chemnitz, D-09107 Chemnitz, Germany

(Dated: April 11, 2003)

\begin{abstract}
Spectroscopic ellipsometry (SE) is used in study GaN films during growth by metal-organic vapor phase epitaxy (MOVPE) and correlation to well known results of plasma-assisted molecular beam epitaxy (PAMBE). Results for the PAMBE reveal clear differences between growth under Ga-rich and N-rich conditions, which are attributed to the presence of a Ga-bilayer on the surface (also seen with low energy electron-diffraction) in the former case. Results for MOVPE surfaces during growth or for surfaces which are stabilized under $\mathrm{NH}_{3}$ are very similar to the N-rich PAMBE result. It is concluded that under normal growth conditions in MOVPE the surface is not terminated by a Ga-bilayer.
\end{abstract}

PACS numbers:

Keywords: GaN; ellipsometry; optical properties; dielectric function 
Surface optical probes have been developed as a very sensitive tool in means of monitoring, and thereby controlling the growth of semiconductor films.$^{1-6}$ In particular, spectroscopic ellipsometry has proven its ability to measure sub-nm thicknesses of roughness or overlayers on surfaces. ${ }^{2,3}$ In case of cubic semiconductors their fourfold bulk symmetry permits the use of reflectance anisotropy spectroscopy measurements in which only the surface contributes to the measurement, ${ }^{5}$ but such measurements are not applicable to the threefold-symmetric (0001) surface of GaN. Using SE one is not limited to any special bulk or surface symmetry for optical characterisation. In particular it is of interest to probe the surface structure and chemistry during GaN growth by metal-organic vapor phase epitaxy (MOVPE) ${ }^{6}$ since the associated high pressures preclude in-situ observation by electron diffraction techniques.

In this work we use spectroscopic ellipsometry to probe the $\mathrm{GaN}(0001)$ surface during growth both by MOVPE and by plasma-assisted molecular beam epitaxy (PAMBE). For the latter case much is already known concerning surface structures during growth, with the results varying as the surface stoichiometry varies from Ga-rich to N-rich. ${ }^{7,8}$ Under Garich conditions it has been established that slightly more than $2 \mathrm{ML}$ (ML $=$ monolayer $=1.14 \times 10^{15}$ atoms $/ \mathrm{cm}^{2}$ ) of Ga resides on the surface during growth, in a metallic fluidlike state. ${ }^{7}$ This Ga bilayer has been shown to have a dramatic influence on the kinetics of the growing surface. ${ }^{8}$ It is therefore of considerable interest to determine whether such a bilayer is also present during GaN growth by MOVPE. In PAMBE ellipsometry reveals clear differences between growth under Ga-rich and N-rich conditions, which are attributed to the presence of the Ga-bilayer on the surface. Results for MOVPE surfaces obtained during growth are found to be practically the same as those obtained from $\mathrm{NH}_{3}$ stabilized surfaces, both of which are very similar to the N-rich PAMBE results. We thus will show that, under normal MOVPE growth conditions, the GaN surface is not terminated by a Ga-bilayer.

The MOVPE growth was performed in a standard horizontal rf heated reactor. At first a GaN nucleation layer was deposited at $550^{\circ} \mathrm{C}$ on the (0001) sapphire substrate. Afterwards the GaN layer of about $1.5 \mu \mathrm{m}$ thickness was grown at an epitaxy temperature of $1040{ }^{\circ} \mathrm{C}$ with trimethylgallium (TMGa) and ammonia precursors at a V/III ratio of 2,000. ${ }^{9}$ The GaN MOVPE on sapphire corresponds to a growth in the (0001) direction with Ga terminated basal plans (Ga-face). ${ }^{10}$ On the base of such a surface we perform Ga-switching experiments at $900^{\circ} \mathrm{C}$ under nitrogen carrier gas. We choose $900^{\circ} \mathrm{C}$ to achieve similar energetic positions 
of the band critical points in the GaN dielectric function for easy comparison to the PAMBE results. Also surface deterioration due to hydrogen etching (from the dissociated ammonia) occurs more slowly than at real growth temperature of $1040^{\circ} \mathrm{C}$ while the general behavior of the surface to precursor switching is proven to be nearly the same.

GaN PAMBE films were deposited on MOVPE grown GaN substrates as described previously and thus their polarity was also Ga-face. The reactive nitrogen was supplied by an rf cracker cell using a flow of approx. $5 \mathrm{sccm} / \mathrm{min}$ molecular nitrogen. The pressure was kept in the range of $8 \times 10^{-5}$ mbar during epitaxy. Ga was supplied via a standard Knudsen evaporation cell. The cell temperature was varied between $910^{\circ} \mathrm{C}$ (low Ga-flux) and $950{ }^{\circ} \mathrm{C}$ (high Ga-flux) which corresponds to a gallium flux ratio of 1:3 estimated from the gallium vapor pressure at $910^{\circ} \mathrm{C}$ and $950^{\circ} \mathrm{C}$, respectively.

Figure 1 shows ellipsometry transients acquired at $4.8 \mathrm{eV}$ during PAMBE growth and during various interrupts in that growth cycle. We plot the imaginary part of the effective dielectric function $\left\langle\varepsilon_{2}\right\rangle$, which describes the dissipative part of the reflected light including overlayer effects like roughness and layer - substrate interferences. We see from Fig. 1 that changes in the surface properties generated by the variations in beam fluxes and growth temperature produce large changes in the value of $\left\langle\varepsilon_{2}\right\rangle$. At a given temperature these changes are reversible when the fluxes are changed back to their original states, indicating that they do not arise simply from roughening of the surface. This is also confirmed by the fact that an increase in $\left\langle\varepsilon_{2}\right\rangle$ here occurs for higher gallium fluxes where AFM has proven a decrease in surface roughness. At the same time low-energy electron diffraction (LEED) observations reveal the presence or absence of the "1 $\times 1 "$ Ga-bilayer on the surface, as shown in Fig. 2(a). In contrast, under N-rich conditions, a $\sqrt{3} \times \sqrt{3}$-R30 $0^{\circ}$ LED pattern is obtained; this pattern has been reported in prior studies of N-rich GaN surfaces. ${ }^{11}$ Surface morphologies after growth under Ga- and N-rich conditions are shown in Figs. 2(c) and (d), respectively. They agree well with prior observations ${ }^{7,8,11,12}$ with the Ga-rich case showing smooth step-flow growth together with Ga droplets on a larger length scale and the N-rich case displaying rough, 3-dimensional growth.

An as measured spectrum of $\left\langle\varepsilon_{2}\right\rangle$, is shown in Fig. 3(a) for PAMBE growth at approx. $850^{\circ} \mathrm{C}$ sample temperature. Here the Ga-rich and N-rich results refer to growth with the Ga effusion cell at $950^{\circ} \mathrm{C}$ or $910^{\circ} \mathrm{C}$, respectively. From the LEED results discussed above we know that the former surface is terminated with a Ga bilayer, and the latter consists of 
some type of N-related reconstruction. Below the band gap energy (about $3 \mathrm{eV}$ at $900{ }^{\circ} \mathrm{C}$ ) the spectra displays Fabry-Perot resonances within the GaN film. Above that energy, the magnitude of $\left\langle\varepsilon_{2}\right\rangle$ is seen to be much larger for the Ga-rich growth compared to the Nrich growth. We attribute this difference purely to the difference in their surface properties (since the changes occur within one second upon varying the growth parameters, as shown in Fig. 1). Using ellipsometric simulations we find that the observed differences in $\left\langle\varepsilon_{2}\right\rangle$ and $\left\langle\varepsilon_{1}\right\rangle$ above the bandgap can be explained through a thin metallic overlayer with a Drude like behavior of free carriers. ${ }^{13}$ A layer thickness of about $0.4 \mathrm{~nm}$ which appears close to the predicted Ga bilayer thickness of $0.48 \mathrm{~nm}^{8}$ accounts for the observed changes in $\langle\varepsilon\rangle$. Further on, the calculated electron density $\left(N_{e} /\left(m_{e f f} / m_{e}\right)\right)$ of $1.2 \times 10^{22} \mathrm{~cm}^{-1}$ corresponds well to the bulk electron density of metals like Ga. By assuming such a metallic layer, significant differences remain only below the fundamental bandgap of GaN. We believe that the influence of the Ga-bilayer on the $\langle\varepsilon\rangle$ spectrum may also include effects of a surface electric field due to a band bending in the upper monolayers or additional interface electronic states. In any case, the high sensitivity of ellipsometry to GaN surface conditions found in Figs. 1 and 3 clearly demonstrates its usefulness as a real-time monitor of GaN surface conditions during growth.

Figure 3(b) shows ellipsometry results for a MOVPE prepared GaN surface, thereby permitting a determination of its surface chemistry. Measurements were made at $900{ }^{\circ} \mathrm{C}$ close to regular MOVPE growth (V/III ratio of 2,000) and during an interruption of that growth when the TMGa source is turned off. This temperature was chosen to achieve similar energetic positions of the bandcritical points in the GaN dielectric function for easy comparison. The behavior at real growth temperature $\left(1050^{\circ} \mathrm{C}\right)$ is quite similar to that at $900^{\circ} \mathrm{C}$ when switching off the Ga source as long as the presence of hydrogen does not etch and roughen the surface. At real growth temperature etching can be observed after some seconds. Apart from slight deviation due to sample variations, the respective results for $\left\langle\varepsilon_{2}\right\rangle$ in Fig.3(b) are nearly identical, indicating that during MOVPE growth the GaN surface is not terminated by a Ga bilayer. Rather, the surface is presumably terminated by a structure involving $\mathrm{N}$ and $\mathrm{Ga}$, and possibly $\mathrm{H}$. This conclusion is supported by noting the magnitude of $\left\langle\varepsilon_{2}\right\rangle$ for the MOVPE results of Fig. 3(b) are quite close to that of the $\mathrm{N}$ rich PAMBE results of Fig. 3(a). Consistent with our interpretation that both surfaces compose of some sort of structure which does not include a metallic Ga-bilayer. Beyond 
this conclusion, we cannot deduce more about the detailed structure of the $\mathrm{NH}_{3}$ stabilized surface from our results. Prior grazing incidence X-ray diffraction measurements (GIXS) have indicated a $\sqrt{3} \times 2 \sqrt{3}$-R30 ${ }^{\circ}$ surface structure during MOVPE growth, ${ }^{14}$ and recent theoretical work provides insight into possible surface structures,${ }^{15}$ but to proof the existence of these structures additional experimental work is needed. This can be done by examining ellipsometric transients during MOVPE growth and / or growth interruptions, respectively. Thereby gallium rich as well as gallium poor surfaces should be obtained.

As noted above, turning off the TMGa source in order to produce an $\mathrm{NH}_{3}$ terminated surface produces very little change in the ellipsometric signal compared to that obtained during regular MOVPE growth conditions. However, starting from an $\mathrm{NH}_{3}$ terminated surface and then attempting to produce a Ga terminated surface by shutting off the $\mathrm{NH}_{3}$ supply and turning on the TMGa supply produces rather complicated results, as shown in Fig. 4. The value of $\left\langle\varepsilon_{2}\right\rangle$ increases rapidly when the TMGa is turned on, reproducibly displaying a small knee in the first second after the turn-on and then continuing to rapidly increase for another 1-2 seconds. The signal then reaches a maximum and begins to decrease exponentially. In the example shown in Fig. 4 the TMGa supply is then turned off and the $\mathrm{NH}_{3}$ supply is turned on after $5 \mathrm{~s}$. Finally the $\left\langle\varepsilon_{2}\right\rangle$ signal approaches a level nearly equal to that at the start of the sequence. If, alternatively, the TMGa supply had been left on the $\left\langle\varepsilon_{2}\right\rangle$ signal seems to approach a constant value of about 3.6 followed by the formation of Ga droplets. The final difference between the $\left\langle\varepsilon_{2}\right\rangle$ signal during TMGa supply and that of the $\mathrm{NH}_{3}$ terminated surface is very close to the difference observed in the PAMBE. But this leads to a non reversible roughening of the surface after switching on again ammonia for stabilization. This is in accordance to the previous finding that smooth surfaces can be grown only under N-rich conditions. We tentatively interpret these results as follows: the initial increase in $\left\langle\varepsilon_{2}\right\rangle$ up to the above-mentioned knee is likely due to the formation of a $\mathrm{Ga}$ rich surface. This is proven by an estimation of the TMGa supply which is in the range of $5 \times 10^{15} \mathrm{Ga}$-atoms $/ \mathrm{s}$ for the used $2 \mu \mathrm{mol} \mathrm{min}^{-1}$. Additional Ga then appears to be deposited on the surface, either in the form of droplets or layers, giving rise to the further increase of $\left\langle\varepsilon_{2}\right\rangle$. However, the complex devolution could be ascribed to the presence of hydrogen and the formation of hydrogen induced reconstructions. ${ }^{15}$ This is also supported by the fact, that the described behavior occurs more distinct but on a larger time scale by using hydrogen as carrier gas. In contrast hydrogen can be neglected in the PAMBE due to the sputter effect 
of the nitrogen plasma.

Finally, in Fig. 3(c) we show spectral results obtained during the MOVPE growth interrupts. The $\mathrm{NH}_{3}$-stabilized surface results are very similar to those for the surface during growth, as noted above. The Ga-pulse results were obtained by measuring the value of $\left\langle\varepsilon_{2}\right\rangle$ observed during transient measurements of the type shown in Fig. 4, and then constructing a spectrum from those values, obtained at certain points after the TMGa flux is turned on. We choose spectra taken at the small knee position as shown in the transient measurements after $\approx 1 \mathrm{~s}$ and after $\approx 8 \mathrm{~s}$ where the TMGa supply is switched off (encircled positions in Fig. 4). The results of $\left\langle\varepsilon_{2}\right\rangle$ are shown by the Ga-pulse spectra in Fig. 3(c). The values of $\left\langle\varepsilon_{2}\right\rangle$ found there are somewhat greater and larger than those for the Ga-rich spectrum of Fig. 3(a), presumably arising from the different surface structures and morphology as discussed above. Finally, the assumed spectra of the stable Ga terminated surface in the MOVPE should be found somewhere in between and the position could be close to that spectra found in the MBE prepared Ga terminated surface.

In summary, we have used spectroscopic ellipsometry to probe the (0001) surface of GaN during growth by MOVPE and PAMBE. Large differences in $\left\langle\varepsilon_{2}\right\rangle$ are found between Garich and N-rich PAMBE results and are attributed to the presence of the surface Ga-bilayer in the former case and its absence in the latter case. Results of surfaces during MOVPE growth or with $\mathrm{NH}_{3}$-stabilisation only are very similar to the N-rich PAMBE spectrum, indicating that the MOVPE surface is not terminated by a Ga bilayer under normal growth conditions. Gallium layers can however be deposited on the MOVPE surface by exposure to TMGa in the absence of $\mathrm{NH}_{3}$, although these layers display a rather complex behavior involving adsorption, roughening and reformation at the growth temperature.

* Electronic address: chrcobet@physik.tu-berlin.de

1 K. Vedam, Thin Solid Films 313-314, 1 (1998).

2 D. E. Aspnes, J. B. Theeten, and F. Hottier, Phys. Rev. B 20, 3292 (1979).

3 D. E. Aspnes, in Optical properties of solids: new developments, edited by B. Seraphin (NorthHolland Publishing Company, Amsterdam, 1975), ISBN 0-7204-0363-4.

4 W. Richter, Appl. Phys. A 75, 129 (2002). 
5 K. Hingerl, D. E. Aspnes, I. Kamiya, and L. T. Florez, Appl. Phys. Lett. 63, 885 (1993).

6 T. Wethkamp, K. Wilmers, N. Esser, W. Richter, O. Ambacher, H. Angerer, G. Jungk, R. L. Johnson, and M. Cardona, Thin Solid Films 313-314, 745 (1998).

7 A. R. Smith, R. M. Feenstra, D. W. Greve, M.-S. Shin, M. Skowronski, J. Neugebauer, and J. Northrup, J. Vac. Sci. Technol. B 16, 2242 (1999).

8 R. M. Feenstra, J. Neugebauer, and J. Northrup, MRS Internet J. Nitride Semicond. Res. 7, 3 (2002).

9 S. Peters, T. Schmidtling, T. Trepk, U. W. Pohl, J.-T. Zettler, and W. Richter, J. Appl. Phys. 88, 4085 (2000).

10 F. A. Ponce, D. P. Bour, W. T. Young, M. Saunders, and J. W. Steeds, Appl. Phys. Lett. 69, 337 (1996).

11 A. Pavlovska and E. Bauer, Surf. Sci. 480, 128 (2001).

12 B. Heying, R. Averbeck, L. F. Chen, E. Haus, H. Riechert, and J. S. Speck, J. Appl. Phys. 88, $1855(2000)$.

13 R. W. Collins and K. Vedam, Encycl. of Appl. Phys. 12, 285 (1995).

14 A. Munkholm, G. B. Stephenson, J. A. Eastman, C. Thompson, P. Fini, J. S. Speck, O. Auciello, P. H. Fuoss, and S. P. DenBaars, Phys. Rev. Lett. 83, 741 (1999).

15 C. G. Van de Walle and J. Neugebauer, Phys. Rev. B 61, 9932 (2000).

\section{Figures}

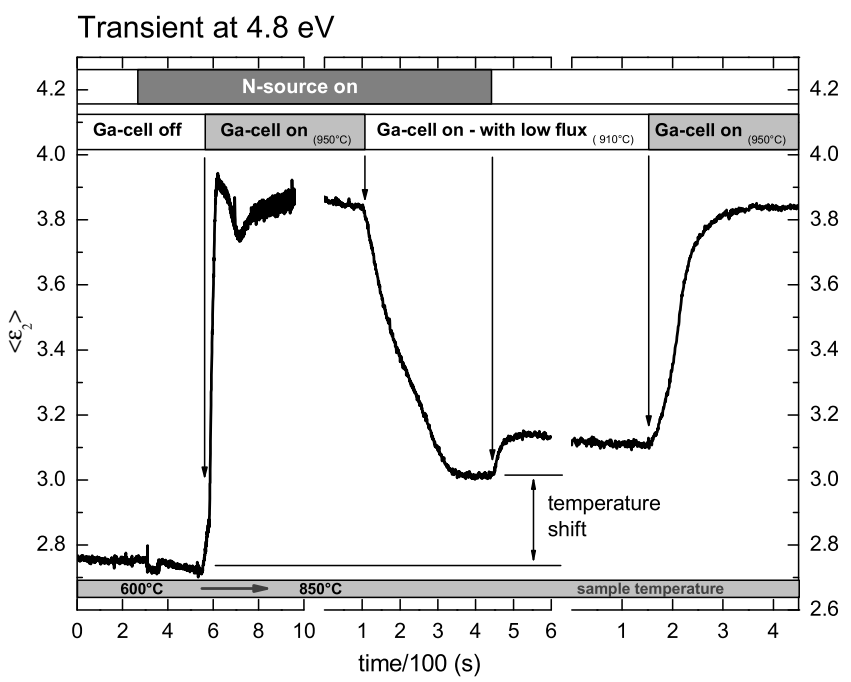


FIG. 1: Transient measurement of the imaginary part of the effective dielectric function, $\left\langle\varepsilon_{2}\right\rangle$, at $4.8 \mathrm{eV}$ during variations in PAMBE growth parameters. First the N-source is turned on. Then the sample temperature is increased from 600 to $850{ }^{\circ} \mathrm{C}$ and simultaneously the Ga shutter is opened with the Ga cell being at $950^{\circ} \mathrm{C}$. The Ga-cell is then ramped down to $910^{\circ} \mathrm{C}$ and then the N-course is turned off. Finally the Ga-cell is ramped back up to $950^{\circ} \mathrm{C}$.
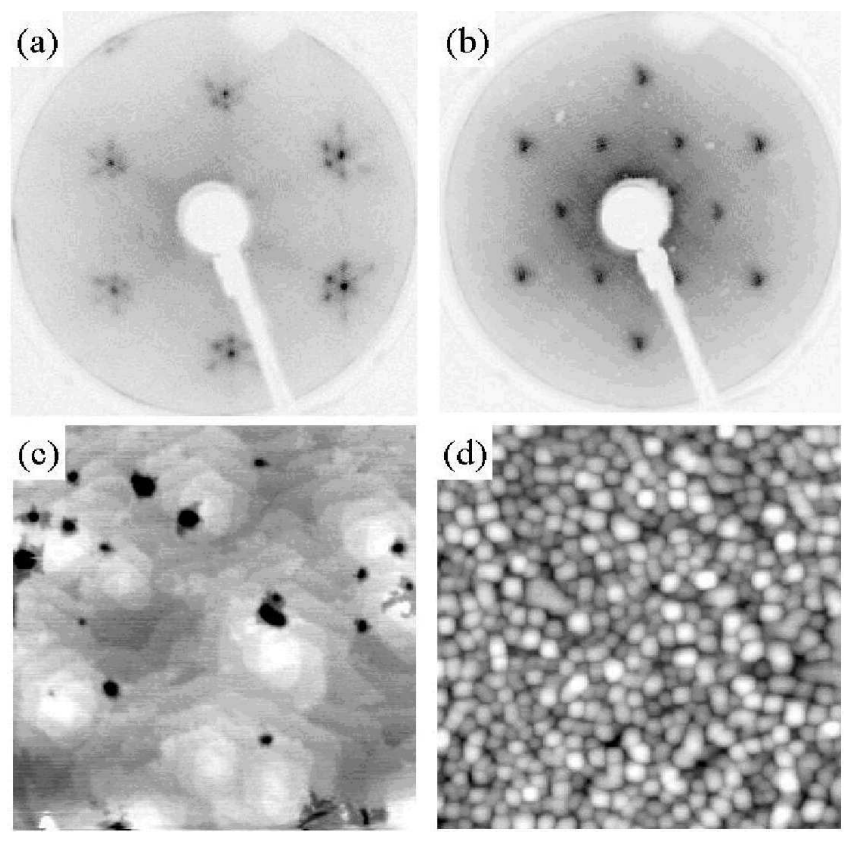

FIG. 2: LEED images obtained from PAMBE surfaces following growth under (a) Ga-rich and (b) N-rich growth conditions, obtained with electron energies of 71 and $63 \mathrm{eV}$, respectively. Atomic force microscopy images from the same surface are shown in (c) and (d), respectively. Both images extend over an area of $1 \times 1 \mu \mathrm{m}^{2}$, and are displayed with gray-scale ranges of 2 and $5 \mathrm{~nm}$, respectively. 


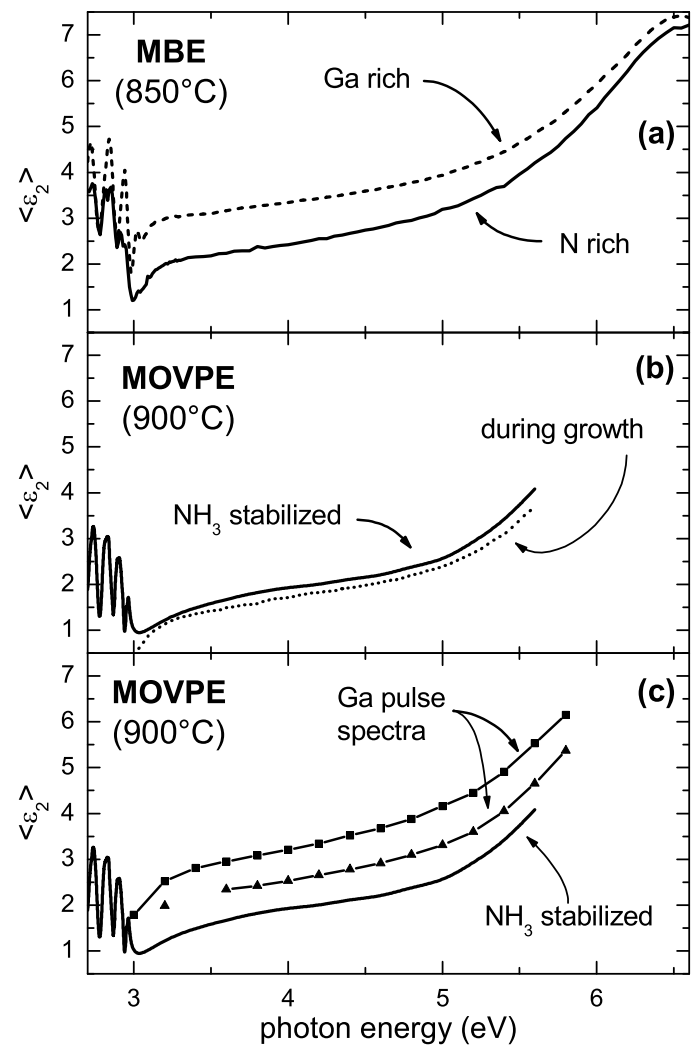

FIG. 3: Imaginary part of the dielectric function $\left\langle\varepsilon_{2}\right\rangle$, for $\mathrm{GaN}(0001)$ films comparing results (a) during PAMBE under Ga-rich or N-rich conditions, (b) during MOVPE growth or stabilized under an $\mathrm{NH}_{3}$-flux, and (c) during MOVPE on $\mathrm{NH}_{3}$-stabilized surfaces or on surfaces exposed to a pulse of TMGa after $\approx 1 \mathrm{~s}$ and $\approx 8 \mathrm{~s}$ in the absence of $\mathrm{NH}_{3}$ (see Fig. 4, encircled positions).

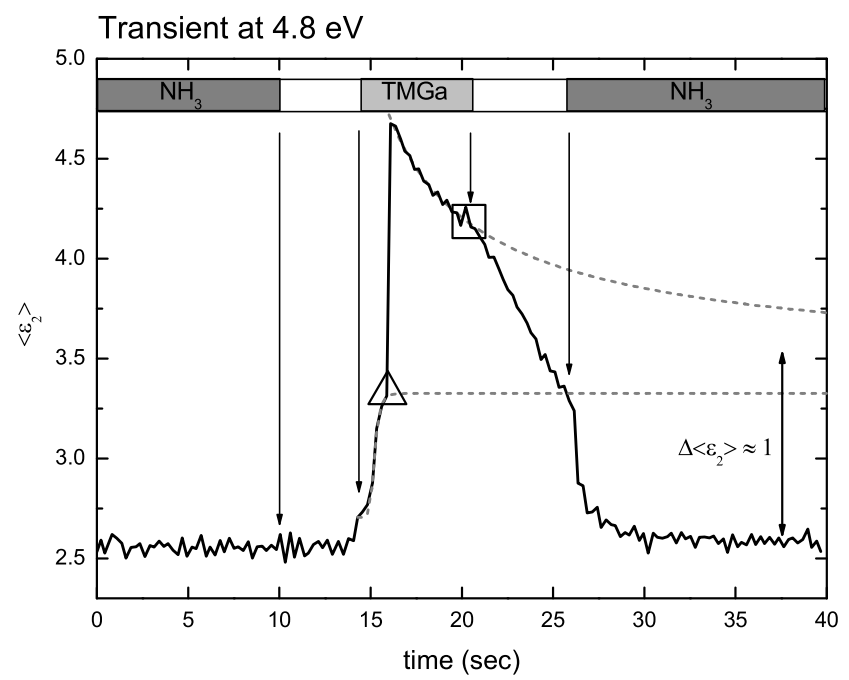


FIG. 4: Transient measurement of imaginary part of the effective dielectric function, $\left\langle\varepsilon_{2}\right\rangle$, at $4.8 \mathrm{eV}$, during variations in MOVPE growth parameter at $900{ }^{\circ} \mathrm{C}$ and using $\mathrm{N}_{2}$ carrier gas. Starting with a surface stabilized under $\mathrm{NH}_{3}$ with no TMGa flux, the $\mathrm{NH}_{3}$ supply is first turned off and $5 \mathrm{~s}$ later the TMGa flux is turned on. A rapid increase in $\left\langle\varepsilon_{2}\right\rangle$ results, displaying a small knee near a value of 3.3 (triangle), increasing to about 4.6, and then exponentially decaying. The TMGa supply was shut off after a duration of $\approx 8 \mathrm{~s}$ (square), and the $\mathrm{NH}_{3}$ was turned on again $5 \mathrm{~s}$ later. The $\left\langle\varepsilon_{2}\right\rangle$ then returns to nearly its original value. 